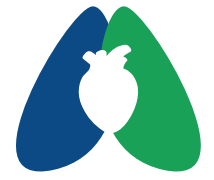

ASSOBRAFIR

C I Ê N C I A
1Programa de Pós-Graduação em Fisioterapia, Departamento de Fisioterapia, Universidade do Estado de Santa Catarina (UDESC), Florianópolis, SC, Brasil.

Como citar: Schivinski CIS. Dispositivos de pressão positiva oscilatória em pacientes com fibrose cística: algumas considerações. ASSOBRAFIR Ciênc. 2020;11:e44121. http://dx.doi.org/10.47066/2177-9333. AC.2020.0025

Submissão em: Julho 13, 2021

Aceito em: Julho 13, 2021

*Autor correspondente: Camila Isabel Santos Schivinski. E-mail: camila.santos@ udesc.br

\section{Dispositivos de pressão positiva oscilatória em pacientes com fibrose cística: algumas considerações}

\author{
Oscillatory positive pressure devices in patients with cystic fibrosis: \\ some considerations
}

Camila Isabel Santos Schivinski ${ }^{1}$ (1)

Promover a independência, o autocuidado e a autogestão do tratamento em indivíduos com fibrose cística (FC) tem sido uma premissa para toda equipe multidisciplinar no manejo da doença ${ }^{1}$, sendo a desobstrução das vias aéreas, 0 suporte nutricional, a reposição de enzimas pancreáticas e a antibioticoterapia crônica os pilares do tratamento ${ }^{2}$ e, sendo assim, o foco constante nas condutas dos respectivos profissionais. Especificamente em relação a fisioterapia respiratória, dispositivos para desobstrução brônquica têm sido desenvolvidos e estão evoluindo cada vez mais ${ }^{3,4}$. Nessa linha, dispositivos oscilatórios que geram oscilações intra ou extratorácicas - por via oral ou externa à parede torácica - se apresentam como alternativas terapêuticas para a rotina dessa população. Nesse contexto, a Cochrane ${ }^{5}$ publicou uma recente atualização referente a revisão do tema, a qual objetivou identificar se os dispositivos oscilatórios - orais ou da parede torácica - são eficazes para a depuração mucociliar, e se são equivalentes ou superiores a outras formas de desobstrução das vias aéreas na FC. Como conclusão, não se constatou superioridade entre os dispositivos.

Historicamente, outras revisões ${ }^{6}$ já apresentaram o resultado da comparação entre dispositivos para desobstrução das vias aéreas e a fisioterapia respiratória padrão (chest physiotherapy - CPT), e a maioria dos estudos não mostra nenhuma vantagem entre as intervenções. No entanto, esses estudos incluídos empregam diferentes intervenções e medidas de resultados e, portanto, não viabilizam metaanálises. Além disso, a maioria das pesquisas que fundamentam essas revisões, foi conduzida há mais de 20 anos, o que compromete a aplicabilidade dos resultados a prática atual, uma vez que o cenário hoje implica em um manejo clínico mais agressivo, melhorias nos antibióticos e novos dispositivos fisioterapêuticos. Especificamente em relação aos dispositivos oscilatórios orais, cujo mecanismo de ação inclui a criação de uma pressão positiva expiratória associada a ondas de vibração oscilatória, as quais evitam o fechamento prematuro das vias aéreas e resultam em mobilização das secreções das vias aéreas, estes apresentam-se como alternativas na terapia, visando a melhora da permeabilidade das vias aéreas e a redução da viscosidade do escarro².

No Brasil, esse tipo de dispositivo se encontra entre o $2^{\circ}$ recurso fisioterapêutico mais recomendado pelas equipes de profissionais dos centros de referência da FC, depois do huffing e seguida de dispositivos com pressão positiva expiratória, além de ser um recurso indicado com maior frequência a partir da idade pré escolar6.

Dessa maneira, ainda não havendo um dispositivo oscilatório considerado padrão ouro no manejo da FC, investigações quanto aos instrumentais de oscilação oral recomendados pelos profissionais na prática clínica, e utilizados por pacientes na rotina terapêutica, se justificam. Com base nisso, o artigo: "Dispositivos de Pressão Positiva Oscilatória em pacientes com Fibrose Cística um estudo de Equivalência" foi publicado pelos autores Francielly D. M. R. do Carmo, Cristiane C. Coelho, Renata C. V. Januário e Evanirso da S. Aquino, na 11ª edição da Assobrafir Ciência de 2020 (https://doi. org/10.47066/2177-9333.AC.2020.0004). 
Trata-se de um estudo cruzado aleatorizado com washout de uma semana, o qual avaliou a equivalência da aplicação de dois dispositivos oscilatórios para desobstrução de vias aéreas na FC - o Shaker ${ }^{\circledR}$ e o Quake®e não identificou diferença significativa comparando-se parâmetros cardiorrespiratórios de frequências cardíaca e respiratória, saturação periférica de oxigênio, peso úmido e seco da secreção brônquica expectorada, entre os dois dispositivos utilizados por oito pacientes. Em relação aos dados basais, houve aumento na frequência respiratória e no peso úmido logo após o uso dos dois dispositivos, sendo que o Quake ${ }^{\circledR}$ foi preferido por sete pacientes, apesar da percepção subjetiva de esforço ter aumentado apenas após a sua realização. Vale recordar que, diferente de dispositivos como o Flutter ${ }^{\circledR}$, o Quake ${ }^{\circledR}$ não é posição e nem fluxo dependente, permite variar os diferenciais de pressão percussiva, e as oscilações da coluna não ocorrem só na expiração, mas também na inspiração. Essa ação nas duas fases da respiração talvez justifique o maior esforço referido com a sua utilização.

Apesar do tamanho amostral limitado, essa pesquisa se mostra relevante, principalmente para realidade do Brasil, uma vez que, com frequência, se discute com pacientes e seus familiares a superioridade entre as intervenções fisioterapêuticas e a orientação profissional quanto ao investimento financeiro para aquisição de um ou outro dispositivo. A equivalência entre esses dois dispositivos também foi avaliada por Suzan et al (2015)e não se constatou diferença entre esses dispositivos em desfechos semelhantes.

Nesse contexto, estudos que investiguem e comparem os efeitos dos dispositivos oscilatórios disponíveis e acessíveis para comunidade, merecem leitura e divulgação. Garantir o autocuidado por meio de recursos que possam ser utilizados de forma independente, e que ainda são práticos, portáteis e de fácil manuseio, como os dois dispositivos oscilatórios analisados no referido estudo de equivalência, têm sido uma indicação em guidelines e publicações na $F^{2-4,6,8}$. Isso porque a promoção da independência, por meio de dispositivos fisioterapêuticos como esses, vai ao encontro do que tem sido estimulado como modelo de assistência multidisciplinar centrada no paciente. Esse modelo inclui a tomada de decisão compartilhada, o controle e a autoeficácia na gestão do tratamento, oportunidades educacionais e vocacionais, capacitação da funcionalidade e da socialização, o que podem levar a um tratamento eficiente e com resultados na qualidade de vida.

Somada a todas essas considerações, a população investigada na referida publicação, com idade mínima de 9 e máxima de 15 anos, caracterizada por pré e adolescentes, é reconhecida com o grupo etário referência para o início dos problemas com a adesão ao tratamento na doença. Com isso, apresentar resultados do uso desses dois dispositivos e a preferência dessa população, contribui para o planejamento da terapia pelo fisioterapeuta, visando a satisfação do paciente e, consequentemente, melhor adesão ao tratamento, o que pode então repercutir em outros parâmetros, como tolerância ao exercício e função respiratória.

Em suma, uma grande variedade de dispositivos oscilatórios orais de desobstrução das vias aéreas está disponível comercialmente, sendo os dois mais recentes o próprio Quake $\AA$ e o Lung Flute ${ }^{\circledR}$. Dessa forma, ainda se faz necessário conhecer e comparar os efeitos desses dispositivos em parâmetros fisiológicos, cardiorrespiratórios, frequência das exacerbações, preferência individual, adesão à terapia e satisfação geral com o tratamento, ainda mais considerando-se aspectos financeiros e a realidade de cada país. É consenso que ensaios clínicos randomizados em longo prazo ainda são necessários, no entanto, a apresentação dos resultados obtidos em pacientes com FC quanto a equivalência entre o Shaker ${ }^{\circledR}$ e o Quake ${ }^{\circledR}$, como na publicação abordada neste editorial, tem sua aplicação. O fato do dispositivo nacional ter apresentado resultados satisfatórios - além do seu menor custo, manuseio fácil e praticidade - endossa sua recomendação por muitos centros de referência do país ${ }^{8}$.

Os profissionais da fisioterapia devem conhecer os mecanismos de ação e efeitos de dispositivos oscilatórios como esses e, respeitando-se a personalização do atendimento na FC, promovê-los no acompanhamento de seus pacientes para gerar autonomia na terapia, uma vez que a autogestão do tratamento fisioterapêutico é uma atividade colaborativa entre o doente e o profissional de saúde.

\section{REFERÊNCIAS}

1. Jamieson N, Fitzgerald D, Singh-Grewal D, Hanson CS, Craig JC, Tong A. Children's experiences of cystic fibrosis: a systematic review of qualitative studies. Pediatrics. 2014 Jun;133(6):e1683-97. http://dx.doi.org/10.1542/peds.20140009. PMid:24843053.

2. Royal Brompton \& Harefield NHS. Clinical Guidelines: Care of Children with Cystic Fibrosis [Internet]. 8th ed. NHS Foundation Trust; 2020 [citado em 2021/07/10. Disponível em: www.rbht.nhs.uk/childrencf.

3. Marks JH. Airway clearance devices in cystic fibrosis. Paediatr Respir Rev. 2007 Mar;8(1):17-23. http://dx.doi.org/10.1016/j. prrv.2007.02.003. PMid:17419974.

4. Osadnik CR, McDonald CF, Holland AE. Advances in airway clearance technologies for chronic obstructive pulmonary disease. Expert Rev Respir Med. 2013;7(6):67385. http://dx.doi.org/10.1586/17476348.2013.847368. PMid:24224510.

5. Morrison L, Milroy S. Oscillating devices for airway clearance in people with cystic fibrosis. Cochrane Database Syst Rev. 2020 Abr 30;4(4):CD006842. https://www.ncbi. nlm.nih.gov/pmc/articles/PMC7197699/. http://dx.doi. org/10.1002/14651858.CD006842.pub5. PMid:32352564.

6. Myers TR. Positive expiratory pressure and oscillatory positive expiratory pressure therapies. Respir Care. 2007 Out;52(10):1308-26. PMid:17894901.

7. Suzan A, Galvão F, Ribeiro A, Ribeiro J, Ribeiro M, Assumpção $M$, et al. Oscilação oral de alta frequência e fibrose cística: 
comparação entre Instrumentais. Conscientiae Saúde. 2015;14(2):283-90. http://dx.doi.org/10.5585/conssaude. v14n2.5418.

8. Donadio MVF, Campos NE, Vendrusculo FM, Stofella AM, Almeida ACS, Ziegler B, et al. Respiratory physical therapy techniques recommended for patients with cystic fibrosis treated in specialized centers. Braz J Phys Ther. 2020 NovDez;24(6):532-8. https://www.ncbi.nlm.nih.gov/pmc/articles/ PMC7779962/. http://dx.doi.org/10.1016/j.bjpt.2019.11.003. PMid:31810864. 